\title{
THE COMPARATIVE ABSORPTION OF VITAMIN A FROM A WATER-MISCIBLE AND AN OILY PREPARATION BY NORMAL HUMAN ADULTS AND PATIENTS WITH STEATORRHEA ${ }^{\perp}$
}

\author{
By BEN C. BARNES, ${ }^{2}$ ERIC E. WOLLAEGER, AND HAROLD L. MASON
}

(From the Divisions of Medicine and Biochemistry, Mayo Foundation and Mayo Clinic, Rochester, Minnesota)

(Submitted for publication November 28, 1949; accepted, April 10, 1950)

Impaired intestinal absorption of vitamin $\mathrm{A}$ in celiac disease was first reported by Chesney and McCoord (1) in 1934. Their evidence for impaired absorption was a minimal rise of vitamin $A$ in serum after the administration of a large dose of the vitamin in oily solution. Other workers (2-6) confirmed this observation in celiac disease and extended it to other types of steatorrhea (cytic fibrosis of the pancreas and sprue).

Recently, aqueous dispersions or water-miscible preparations of vitamin A have become available. Comparative studies indicate that the oral administration of such preparations to normal human infants and experimental animals results in a greater rise in the concentration of vitamin A in the plasma and in a lesser loss of the vitamin in the feces than does the administration of an equal dose of vitamin $\mathrm{A}$ in oily solution (7).

The purpose of the present study was to determine whether vitamin $\mathrm{A}$ in a water-miscible preparation would be more efficiently absorbed than that in an oily medium by patients with steatorrhea (particularly nontropical sprue). During the course of our investigation, Kramer and co-workers (8) reported higher serum levels of vitamin $\mathrm{A}$ in celiac disease following the administration of an aqueous dispersion than when vitamin $A$ in oil was given perorally. Others $(9,10)$ confirmed these results in celiac disease and obtained similar results in hepatic disease and cystic fibrosis of the pancreas. Recently Fox (11) reported that eight patients with active sprue showed very poor absorption when fed unemulsified vitamin A. When these same patients were fed

1 Abridgment of thesis submitted by Dr. Barnes to the Faculty of the Graduate School of the University of Minnesota in partial fulfillment of the requirements for the degree of Master of Science in Medicine.

2 Fellow in Medicine, Mayo Foundation. emulsified material, the absorption of vitamin A was increased to normal in every case except one. No fecal excretion studies were done in conjunction with these or previous studies of vitamin A absorption in patients with steatorrhea. Since determination of blood levels alone might give an erroneous conception concerning vitamin A absorption, it seemed important to measure concurrently the blood concentration and fecal losses of vitamin A after the administration of test doses by mouth. The studies of Kagan, Thomas, Jordan, and Abt (12) indicate that plasma levels of vitamin A following the administration of a large test dose by mouth, may be considerably influenced by factors other than intestinal absorption.

\section{METHOD OF STUDY}

The vitamin A tolerance test ${ }^{3}$ combined with fecal and urinary excretion of the vitamin was employed as the means of studying vitamin A absorption. Two preparations of vitamin A were available for comparison. The water-miscible preparation ${ }^{4}$ contained vitamin A alcohol, 37,000 U. S. P. units per cc., made soluble with 40 volumes per cent of Tween number 20 , a polyoxyalkylene derivative of sorbitan monolaurate. Tween number 20 is a surface tension depressant or wetting agent. Studies on rats and monkeys over a long period have revealed it to be nontoxic (13). The other preparation of vitamin A was a concentrate of natural esters in fish liver oil which was administered in capsules containing 50,000 U. S. P. units each.

Studies were carried out on 12 control subjects ${ }^{5}$ and

3 The term "vitamin A tolerance test" has been commonly used in the literature to indicate the response of plasma vitamin $A$ level to an orally administered test dose of vitamin A. We have used the term in this same manner.

4 The water-miscible preparation was generously furnished through the courtesy of Dr. Samuel Gordon, Endo Products, Richmond Hill, New York.

5 Dr. Magnus A. Peterson, Superintendent of the Rochester (Minnesota) State Hospital, kindly permitted us to study these subjects. 
six patients with steatorrhea. The 12 control subjects were cooperative mental patients who were physically healthy except for one patient who had moderately severe diabetes mellitus controlled by diet and insulin. Five of the six patients with steatorrhea had nontropical sprue. They presented the symptoms characteristic of this disease, such as fatty diarrhea, flatulence, glossitis, loss of weight, weakness, tetany, edema and hemorrhage (due to hypoprothrombinemia). Characteristic abnormalities revealed by laboratory tests were also present, such as macrocytic anemia, hypoproteinemia, hypoprothrombinemia, hypocalcemia, osteoporosis, deficiency pattern in the small intestine and flat glucose tolerance curve. The sixth patient had steatorrhea associated with severe diabetes mellitus complicated by diabetic retinopathy and neuropathy. That the steatorrhea in this case was not due to insufficiency of external pancreatic secretion was demonstrated by the finding of normal values for volume, bicarbonate and pancreatic enzymes of the duodenal contents following stimulation with secretin. In all six cases the presence of steatorrhea was shown by the finding of more than $10 \mathrm{gm}$. of fecal lipid per 24 hours while the patients were taking a diet containing $100 \mathrm{gm}$. or less of fat per day. In a study of 10 normal persons taking a test diet containing $101 \mathrm{gm}$. of fat per day, Wollaeger, Comfort, and Osterberg (14) found an average excretion of $4.1 \mathrm{gm}$. of fecal lipid per day and no excretion greater than $7 \mathrm{gm}$. per day. Four of our patients excreted more than $20 \mathrm{gm}$. per day and the other two excreted 14 and $18 \mathrm{gm}$. per day, respectively. Since all six patients were passing several grossly fatty stools daily, prolonged studies were not considered necessary to demonstrate the presence of steatorrhea. Nevertheless, such prolonged studies (periods of six days or longer) were carried out in two cases. In the other four cases stools were collected over periods varying from 24 to 72 hours.

Two vitamin A tolerance tests, performed 72 hours apart, were carried out on each subject studied. One test was done with the water-miscible preparation and the other with an equal dose of vitamin $\mathrm{A}$ in an oily medium. The test dose was 7,500 I. U. (U. S. P. units) per kilogram of body weight. Plasma levels and fecal and urinary losses of vitamin $\mathrm{A}$ were determined before and for a period of 72 hours after each test dose was administered. Half of the 12 controls and half of the six patients with steatorrhea received the water-miscible preparation for the first test and the oil solution for the second test. In the remaining controls and patients this order was reversed.

All of the controls and patients received preliminary doses of vitamin A for a period of at least four days before beginning the vitamin A tolerance tests. The daily dose during this preliminary period was $60,000 \mathrm{I}$. U. of vitamin A given in the form of a water-miscible, multivitamin preparation. ${ }^{6}$ This was done in order to insure

\footnotetext{
6 Known as "Vifort" and donated through the courtesy of Dr. Samuel Gordon, Endo Products, Richmond Hill, New York.
}

the presence of adequate stores of vitamin $\mathrm{A}$ in the tissues which might previously have been depleted because of inadequate intake or impaired absorption.

The relationship between the amount of vitamin $A$ present in the tissues and the level to which the concentration of the vitamin will rise in the plasma after administration of a large oral dose is not entirely clear. However, it is conceivable that vitamin A may be very rapidly withdrawn from the blood when tissue stores are low and thus a low tolerance curve would result even when absorption of vitamin A from the gastrointestinal tract has been normal. The studies of Josephs (15) with rats suggest that the level of hepatic stores may influence the height of the postabsorptive rise of serum vitamin $A$ when large doses are used. He hypothesized that the tissues become "saturated" with vitamin A and are unable to take up more vitamin $A$ as fast as it is supplied to them. The absorbed vitamin A then "piles up" in the serum to some extent. The work of Ruch and associates (16) on skin disorders related to vitamin A deficiency described a case of congenital dyskeratosis in which two vitamin A tolerance tests separated by a fiveday interval were carried out. In the first test only a slight rise in the serum vitamin A was obtained. During the second test the serum vitamin A rose to high levels. Fecal excretion of the vitamin was essentially the same following both tests. These results were interpreted as indicating that the massive dose administered during the first tolerance test filled up depleted hepatic stores. The liver was then well "saturated" during the second test, allowing the serum vitamin $A$ to rise considerably. In dermatologic and laboratory experience with the vitamin A tolerance test at the Mayo Clinic this phenomenon has been repeatedly observed. Four of the controls in Breese and McCoord's (17) studies on celiac disease with the vitamin A tolerance test showed initially flat serum curves. This fact was interpreted as indicating that the controls had "recovered the normal power to absorb vitamin A." In the absence of fecal studies such a statement does not seem warranted. It would seem quite probable that larger tissue stores of vitamin A contributed to the higher serum levels obtained during the second tests.

$V$ itamin $A$ determinations. A sample of venous blood for vitamin A determination was drawn on the day following the last dose of the preliminary vitamin $\mathbf{A}$ administration, and just prior to the ingestion of the test dose. This pretest level served both as a base line for succeeding determinations following the test dose and as some indication of vitamin A storage according to the observations of Popper and his co-workers (18). ${ }^{7}$ After oral administration of the test dose venous blood samples were taken at two, four, six, nine, 24 and 48 hours for determination of vitamin $A$ in the plasma. Seventy-two hours after the first oral test dose another plasma vitamin A level

7 Popper and his co-workers found human blood serum levels of 60 micrograms (approximately $196 \mathrm{I}$. U.) or more of vitamin A per $100 \mathrm{cc}$. associated with at least 70 micrograms of vitamin A per $\mathrm{gm}$. of liver tissue. The hepatic values were considered high for storage levels. 
was obtained and immediately following this the second vitamin A tolerance test was done in the same manner.

Twenty-four-hour collections of feces were begun the morning prior to the first test dose and were analyzed daily for vitamin A until 72 hours after the second test dose. Urinary collections and analyses were done in the same way.

A modification (19) of McCoord and Luce-Clausen's (20) method for determining carotene and vitamin A was used. The Coleman junior spectrophotometer was calibrated with crystalline $\beta$-carotene and vitamin $\mathrm{A}$ of known potency.

\section{RESULTS}

Plasma, fecal and urinary carotene. Among the controls, plasma carotene values ranged from 32 to 204 micrograms per $100 \mathrm{cc}$. with a mean of 79.3. The five patients with nontropical sprue had low levels of plasma carotene, the range of the mean values being 3.4 to 15.7 micrograms per $100 \mathrm{cc}$. of plasma. The patient with steatorrhea and diabetes mellitus had a normal level of plasma carotene, the mean value being 65.6 micrograms per $100 \mathrm{cc}$. of plasma.

The presence of other pigments in the feces made the values for fecal carotene somewhat uncertain. Consequently these values are not presented. Nevertheless, the values for vitamin A were corrected for the apparent amount of carotene, since it was considered a sounder procedure to assume that all the pigment was carotene than to make no correction at all. In any event the corrections were relatively small. There was no significant excretion of carotene in the urine.

Pretest levels of plasma vitamin $A$. After the preliminary four-day period of fortification with water-miscible vitamin $A$, the pretest values for plasma vitamin $\mathrm{A}$ in controls and patients were within normal limits. Among the controls the range was 120 to $261 \mathrm{I}$. U. per $100 \mathrm{cc}$. of plasma, mean $182 \pm 13.6$. The range in the case of the patients was approximately the same, 104 to 202 I. U. per $100 \mathrm{cc}$. of plasma, mean $147 \pm 14.9$. Judging from the work of Popper and his associates (18) the controls and patients had adequate hepatic stores of vitamin A prior to the vitamin A tolerance tests.

Plasma vitamin $A$ levels after administration of test doses. In the majority of the controls the levels of plasma vitamin A rose more rapidly and to a greater height after administration of the water-miscible preparation of vitamin A than when

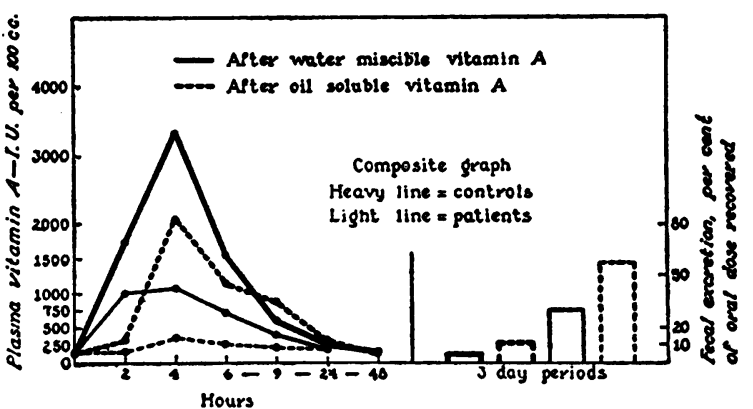

Fig. 1. Comparative Absorption of Vitamin A from OIly aNd Aqueous Preparations Determined by Concurrent Measurement of Plasma Vitamin A Levels and Fecal Losses after Administration of Standardized Test Doses by Mouth

vitamin $A$ in oil solution was ingested (Figure 1 and Table I). All of the patients with steatorrhea had flat plasma curves of vitamin A levels after peroral administration of the oil solution of vita$\min \mathrm{A}$.

Plasma curves after administration of watermiscible vitamin A were distinctly higher in each patient than the corresponding curves after administration of vitamin $\mathrm{A}$ in oil. However, the five patients with nontropical sprue had abnormally low curves after administration of watermiscible vitamin A when compared with corresponding curves in the controls. This finding is in distinction to that of Fox (11) who reported normal plasma curves in seven of eight patients with sprue when an emulsified preparation of

TABLE I

Maximal increase of plasma vitamin A, I.U. per 100 cc. after peroral administration of aqueous and oily preparations

\begin{tabular}{l|c|c|c}
\hline \hline & Mean & $\begin{array}{c}\text { Standard } \\
\text { deviation } \\
\text { of mean }\end{array}$ & Range \\
\hline \multicolumn{3}{c|}{ Controls } \\
\hline \begin{tabular}{l|c|c|} 
After water-miscible \\
vitamin A
\end{tabular} & 3,179 & 295 & $1,612-4,923$ \\
\hline $\begin{array}{c}\text { After oil-soluble } \\
\text { vitamin A }\end{array}$ & 2,193 & 353 & $876-4,313$ \\
\hline
\end{tabular}

Patients

\begin{tabular}{l|c|c|c}
\hline $\begin{array}{c}\text { After water-miscible } \\
\text { vitamin A }\end{array}$ & 1,160 & 128 & $346-3,458$ \\
\hline $\begin{array}{c}\text { After oil-soluble } \\
\text { vitamin A }\end{array}$ & 228 & 42 & $110-509$ \\
\hline
\end{tabular}


TABLE II

Fecal excretion of vitamin $A$ after peroral administration of aqueous and oily preparations, percentage of oral dose

\begin{tabular}{l|c|c|c}
\hline & Mean & $\begin{array}{c}\text { Standard } \\
\text { deviation } \\
\text { of mean }\end{array}$ & Range \\
\hline $\begin{array}{l}\text { Controls } \\
\begin{array}{l}\text { After water-miscible } \\
\text { vitamin A }\end{array}\end{array}$ & 4.8 & 1.8 & $0.0-24.4$ \\
\hline $\begin{array}{l}\text { After oil-soluble } \\
\text { vitamin A }\end{array}$ & 12.2 & 3.4 & $0.3-49.5$ \\
\hline $\begin{array}{l}\text { After water-miscible } \\
\text { vitamin A }\end{array}$ & Patients & 8.2 & $14.3-71.0$ \\
\hline $\begin{array}{l}\text { After oil-soluble } \\
\text { vitamin A }\end{array}$ & 56.6 & 7.5 & $31.1-80.2$ \\
\hline
\end{tabular}

vitamin A was administered. The patient with diabetes mellitus and steatorrhea had a normal plasma curve after ingestion of the water-miscible preparation.

Fecal excretion of vitamin $A$. Fecal excretion is expressed as the percentage of the oral dose of vitamin A recovered in the stools during the threeday period following the test dose. Fecal losses of vitamin A were negligible after 48 to 72 hours.

The mean fecal loss of vitamin $\mathrm{A}$ in the controls was more than twice as much after administration of the oil solution of vitamin $\mathrm{A}$ as the loss after ingestion of the water-miscible preparation (Table II). The quantity of vitamin A excreted in the stools after peroral administration of the water-miscible product is comparable with that reported by Lewis and associates (7) in experimental animals and healthy infants. The value obtained with the oily preparation is less than that reported by these same workers.

Average fecal losses in the patients with steatorrhea were excessive after administration of both preparations of vitamin A. There was significantly less vitamin A lost in the stools after the peroral administration of the water-miscible preparation than when the oily solution was ingested.

The vitamin A absorption studies in the patient with diabetes mellitus and steatorrhea emphasize the importance of measuring fecal losses of the vitamin. In this particular case a normal plasma curve and excessive fecal excretion of vitamin A
(71 per cent of the dose ingested) resulted from the oral administration of the water-miscible preparation of vitamin A. Had not fecal losses of vitamin $A$ been determined in this instance, the erroneous conclusion might have been drawn that normal intestinal absorption of vitamin $A$ had taken place.

In order to compare the relative absorbabilities of the oily solution and water-miscible preparation of vitamin $A$ in controls and patients, the mean values for plasma vitamin $A$ levels and for fecal excretion were plotted in the form of a composite graph (Figure 1).

Urinary excretion of vitamin $A$. No significant loss of vitamin A occurred in the urine even in the face of large oral doses and high plasma concentrations (in controls). This agrees with observations of Ruch and his colleagues (16).

\section{COMMENT}

In attempting to explain the more rapid and more complete absorption of the water-miscible preparation as compared with the oily solution of vitamin A among the controls, two factors seem important. First, because of the fine particle size of vitamin $\mathrm{A}$ in the aqueous dispersion $(8,9)$ further emulsification within the intestinal lumen was probably not necessary. Second, vitamin A in the water-miscible preparation was in the form of the free alcohol and, therefore, hydrolysis within the lumen (21) of the bowel was also not necessary. Both factors would tend to enhance the speed and completeness of vitamin A absorption in the water-miscible preparation.

Why should there be an abnormally retarded and incomplete absorption of both preparations of vitamin $\mathrm{A}$ in the patients with nontropical sprue? In the light of present knowledge concerning vitamin A absorption and nontropical sprue, this question cannot be answered definitely. There is a good deal of evidence to show that there is impaired intestinal absorption of water-soluble as well as fatty food material in patients with this disease. Such evidence includes increased fecal nitrogen losses (22), flat oral glucose tolerance curves, normal intravenous glucose tolerance tests, and signs of deficiencies of water-soluble vitamins. Impaired motility of the intestinal tract as demonstrated by roentgen examination probably is a factor in the malabsorption of nutritive substances 
in sprue $(23,24)$. The retarded absorption of the finely emulsified water-miscible vitamin A alcohol in the cases of nontropical sprue suggests that there may be a delay in passage of the vitamin across the intestinal wall, since the vitamin within the lumen of the bowel is apparently in an optimal state for absorption.

The patient with diabetes mellitus and steatorrhea differed from the patients with nontropical sprue in regard to vitamin A absorption. A normal plasma curve after administration of the aqueous preparation and a flat curve with the oily solution of vitamin were obtained, with excessive fecal losses of the vitamin after administration of both preparations. There was roentgenographic evidence of hypermotility of the small bowel in this patient which may have been a factor in the impaired vitamin $A$ absorption.

Although flat plasma vitamin A curves usually indicate impaired intestinal absorption, they may occasionally be found in persons with normal absorption, as Ruch has pointed out. Conversely, it is possible to obtain a normal vitamin A tolerance curve in some patients with markedly impaired absorption, as in our case with diabetes and steatorrhea when the water-miscible preparation was administered. In such instances errors of interpretation can be prevented only by measuring fecal vitamin A losses. In order to avoid the influence of depleted vitamin A reserves the vitamin A tolerance test should be performed after preliminary dosage with vitamin $\mathrm{A}$.

\section{SUM MARY}

The gastro-intestinal absorbabilities of an oily solution and an aqueous dispersion of vitamin $\mathrm{A}$ were compared in 12 adult control subjects and six patients with steatorrhea. The steatorrhea in five patients was a manifestation of nontropical sprue. The remaining patient had steatorrhea associated with diabetes mellitus.

Vitamin A absorption was studied by performing two vitamin $\mathrm{A}$ tolerance tests combined with measurement of fecal and urinary vitamin A losses in each subject. One tolerance test was done using the oily preparation and the other employing the aqueous dispersion of vitamin $A$. The controls and patients received preliminary dosage with the water-miscible preparation of vitamin A.

The controls displayed higher plasma curves and lower fecal losses of vitamin $\mathrm{A}$ after ingestion of the aqueous dispersion than after taking the oily preparation of vitamin A. The patients with nontropical sprue had flat plasma curves and excessive fecal excretion of vitamin A with both preparations. However, the vitamin $\mathrm{A}$ in the aqueous dispersion was absorbed more rapidly and less of it was excreted in the feces than was the vitamin in oily solution. One patient with diabetes mellitus and steatorrhea had a flat plasma curve after ingestion of the oily material, a normal curve with the aqueous dispersion, and excessive fecal losses of vitamin A with both preparations. Evidence is presented to show that the vitamin A tolerance curve is not always an accurate measure of vitamin A absorption from the intestine. There is reason to believe that it becomes more consistently accurate in this regard: 1) when preliminary doses of vitamin $\mathrm{A}$ are administered to insure adequate tissue storage at the time the test is performed, and 2) when measurement of fecal losses of vita$\min A$ is carried out concomitantly.

\section{BIBLIOGRAPHY}

1. Chesney, Jack, and McCoord, A. B., Vitamin A of serum following administration of haliver oil in normal children and in chronic steatorrhea. Proc. Soc. Exper. Biol. \& Med., 1934, 31, 887.

2. Adlersberg, David, and Sobotka, Harry, Fat and vita$\min A$ absorption in sprue and jejunoileitis. Gastroenterology, 1943, 1, 357.

3. Blackfan, K. D., and May, C. D., Inspissation of secretion, dilatation of the ducts and acini, atrophy and fibrosis of the pancreas in infants. J. Pediat., 1938, 13, 627.

4. Darby, W. J., Kaser, M. M., and Jones, E., Influence of pteroylglutamic acid (member of vitamin $\mathbf{M}$ group) on absorption of vitamin $\mathbf{A}$ and carotene by patients with sprue. J. Nutrition, 1947, 33, 243.

5. May, C. D., and McCreary, J. F., The absorption of vitamin A in celiac disease. J. Pediat., 1941, 18, 200.

6. May, C. D., Blackfan, K. D., McCreary, J. F., and Allen, F. H., Jr., Clinical studies of vitamin A in infants and in children. Am. J. Dis. Child., 1940, 59, 1167.

7. Lewis, J. M., Bodansky, O., Birmingham, J., and Cohlan, S. Q., Comparative absorption, excretion and storage of oily and aqueous preparations of vitamin A. J. Pediat., 1947, 31, 496.

8. Kramer, Benjamin, Sobel, A. E., and Gottfried, S. P., Serum levels of vitamin A in children: a comparison following the oral and intramuscular administration of vitamin $A$ in oily and in aqueous mediums. Am. J. Dis. Child., 1947, 73, 543. 
9. May, C. D., and Lowe, C. U., The absorption of orally administered emulsified lipid in normal children and in children with steatorrhea. J. Clin. Invest., 1948, $27,226$.

10. Popper, Hans, Steigmann, Frederick, and, Dyniewicz, H. A., Treatment of endogenous hypovitaminemia $\mathrm{A}$ in liver disease. Proc. Central Soc. Clin. Research, 1947, 20, 12.

11. Fox, H. J., Absorption of unemulsified and emulsified vitamin A in sprue. J. Lab. \& Clin. Med., 1949, 34, 1140.

12. Kagan, B. M., Thomas, E. M., Jordon, D. A., and Abt, A. F., Serum vitamin A and total plasma lipid concentrations as influenced by the oral administration of vitamin $A$ to children with the nephrotic syndrome. J. Clin. Invest., 1950, 29, 141.

13. Krantz, J. C., Jr., Personal communication.

14. Wollaeger, E. E., Comfort, M. W., and Osterberg, A. E., Total solids, fat and nitrogen in the feces. III. A study of normal persons taking a test diet containing a moderate amount of fat; comparison with results obtained with normal persons taking a test diet containing a large amount of fat. Gastroenterology, 1947, 9, 272.

15a. Josephs, H. W., Factors influencing the level of vita$\min A$ in the blood of rats. Bull. Johns Hopkins Hosp., 1942, 71, 253.

b. Josephs, H. W., Studies in vitamin A: Influence of vitamin $A$ on serum lipids of normal and deficient rats. Ibid., 1942, 71, 265.
16. Ruch, D. M., Brunsting, L. A., and Osterberg, A. E., Use of vitamin A tolerance test in certain cases of dermatologic disorders. Proc. Staff Meet., Mayo Clin., 1946, 21, 209.

17. Breese, B. B., Jr., and McCoord, A. B., Vitamin A absorption in celiac disease. J. Pediat., 1939, 15, 183.

18. Popper, Hans, Steigmann, Frederick, Mayer, K. A., and Zevin, S. S., Relation between hepatic and plasma concentrations of vitamin $A$ in human beings. Arch. Int. Med., 1943, 72, 439.

19. Ruch, D. M., Vitamin A studies in certain cutaneous disorders. Thesis for M.S. degree submitted to Graduate School of University of Minnesota, 1945, $56 \mathrm{pp}$.

20. McCoord, A. B., and Luce-Clausen, E. M., The storage of vitamin $A$ in the liver of the rat. J. Nutrition, 1934, 7, 557.

21. Gray, E. L., Morgareidge, Kenneth, and Cawley, J. D., Intestinal absorption of vitamin $\mathrm{A}$ in the normal rat. J. Nutrition, 1940, $20,67$.

22. Wollaeger, E. E., and Comfort, M. W., Personal communication.

23. Flax, L. J., Barnes, M., and Reichert, L. J., Vita$\min \mathrm{A}$ absorption and its relation to intestinal motility in fibrocystic disease of the pancreas. $\mathrm{J}$. Pediat., 1942, 21, 475.

24. Ingelfinger, F. J., Moss, R. E., and Helm, J. D., Jr., Effect of atropine upon absorption of vitamin A. J. Clin. Invest., 1943, 22, 699. 